\title{
Selective Refinement Network for High Performance Face Detection
}

\author{
Cheng Chi, ${ }^{1,3 *}$ Shifeng Zhang, ${ }^{2,3 * \dagger}$ Junliang Xing, ${ }^{2,3}$ Zhen Lei, $^{2,3}$ Stan Z. Li, ${ }^{2,3}$ Xudong Zou ${ }^{1,3}$ \\ ${ }^{1}$ Institute of Electronics, Chinese Academy of Sciences, Beijing, China \\ ${ }^{2}$ CBSR \& NLPR, Institute of Automation, Chinese Academy of Sciences, Beijing, China \\ ${ }^{3}$ University of Chinese Academy of Sciences, Beijing, China
}

\begin{abstract}
High performance face detection remains a very challenging problem, especially when there exists many tiny faces. This paper presents a novel single-shot face detector, named Selective Refinement Network (SRN), which introduces novel twostep classification and regression operations selectively into an anchor-based face detector to reduce false positives and improve location accuracy simultaneously. In particular, the SRN consists of two modules: the Selective Two-step Classification (STC) module and the Selective Two-step Regression (STR) module. The STC aims to filter out most simple negative anchors from low level detection layers to reduce the search space for the subsequent classifier, while the STR is designed to coarsely adjust the locations and sizes of anchors from high level detection layers to provide better initialization for the subsequent regressor. Moreover, we design a Receptive Field Enhancement (RFE) block to provide more diverse receptive field, which helps to better capture faces in some extreme poses. As a consequence, the proposed SRN detector achieves state-of-the-art performance on all the widely used face detection benchmarks, including AFW, PASCAL face, FDDB, and WIDER FACE datasets. Codes will be released to facilitate further studies on the face detection problem.
\end{abstract}

\section{Introduction}

Face detection is a long-standing problem in computer vision with extensive applications including face alignment, face analysis, face recognition, etc. Starting from the pioneering work of Viola-Jones (Viola and Jones 2004), face detection has made great progress. The performances on several well-known datasets have been improved consistently, even tend to be saturated. To further improve the performance of face detection has become a challenging issue. In our opinion, there remains room for improvement in two aspects: (a) recall efficiency: number of false positives needs to be reduced at the high recall rates; (b) location accuracy: accuracy of the bounding box location needs to be improved. These two problems are elaborated as follows.

On the one hand, the average precision (AP) of current face detection algorithms is already very high, but the precision is not high enough at high recall rates, e.g., as shown

\footnotetext{
*These authors contributed equally to this work.

${ }^{\dagger}$ Corresponding author

Copyright (C) 2019, Association for the Advancement of Artificial Intelligence (www.aaai.org). All rights reserved.
}

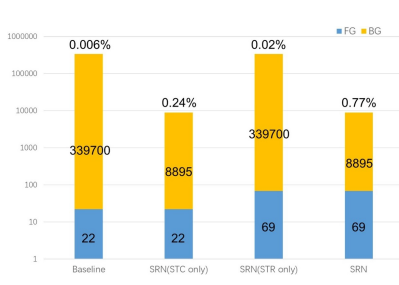

(a) Effect on Class Imbalance

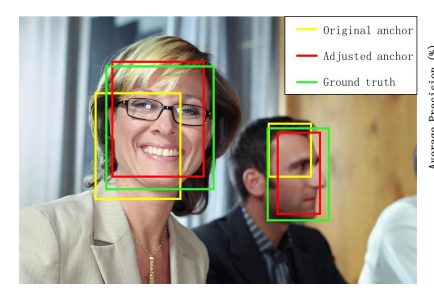

(c) Adjusted Anchor

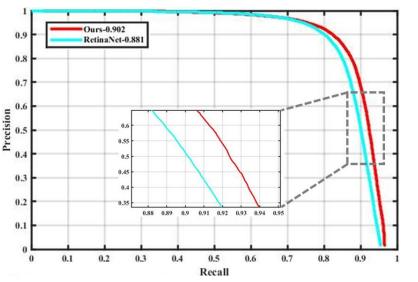

(b) Recall Efficiency

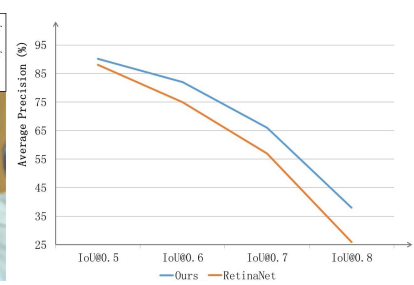

(d) Location Accuracy
Figure 1: The effects of STC and STR on recall efficiency and location accuracy. (a) The STC and STR increase the positives/negatives ratio by about 38 and 3 times respectively, (b) which improve the precision by about $20 \%$ at high recall rates. (c) The STR provides better initialization for the subsequent regressor, (d) which produces more accurate locations, i.e., as the IoU threshold increases, the AP gap gradually increases.

in Figure 1(b) of RetinaNet (Lin et al. 2017b), the precision is only about $50 \%$ (half of detections are false positives) when the recall rate is equal to $90 \%$, which we define as the low recall efficiency. Reflected on the shape of the Precision-Recall curve, it has extended far enough to the right, but not steep enough. The reason is that existing algorithms pay more attention to pursuing high recall rate but ignore the problem of excessive false positives. Analyzing with anchor-based face detectors, they detect faces by classifying and regressing a series of preset anchors, which are generated by regularly tiling a collection of boxes with different scales and aspect ratios. To detect the tiny faces, e.g., less than $16 \times 16$ pixels, it is necessary to tile plenty of small anchors over the image. This can improve the recall rate yet cause the extreme class imbalance problem, which is the culprit leading to excessive 
false positives. To address this issue, researchers propose several solutions. R-CNN-like detectors (Girshick 2015; Ren et al. 2017) address the class imbalance by a two-stage cascade and sampling heuristics. As for single-shot detectors, RetinaNet proposes the focal loss to focus training on a sparse set of hard examples and down-weight the loss assigned to well-classified examples. RefineDet (Zhang et al. 2018) addresses this issue using a preset threshold to filter out negative anchors. However, RetinaNet takes all the samples into account, which also leads to quite a few false positives. Although RefineDet filters out a large number of simple negative samples, it uses hard negative mining in both two steps, and does not make full use of negative samples. Thus, the recall efficiency of them both can be improved.

On the other hand, the location accuracy in the face detection task is gradually attracting the attention of researchers. Although current evaluation criteria of most face detection datasets (Jain and Learned-Miller 2010; Yang et al. 2016) do not focus on the location accuracy, the WIDER Face Challenge $^{1}$ adopts MS COCO (Lin et al. 2014) evaluation criterion, which puts more emphasis on bounding box location accuracy. To visualize this issue, we use different IoU thresholds to evaluate our trained face detector based on RetinaNet on the WIDER FACE dataset. As shown in Figure 1(d), as the IoU threshold increases, the AP drops dramatically, indicating that the accuracy of the bounding box location needs to be improved. To this end, Gidaris et al. (Gidaris and Komodakis 2015) propose iterative regression during inference to improve the accuracy. Cascade R-CNN (Cai and Vasconcelos 2018) addresses this issue by cascading R-CNN with different IoU thresholds. RefineDet (Zhang et al. 2018) applies two-step regression to single-shot detector. However, blindly adding multi-step regression to the specific task (i.e., face detection) is often counterproductive.

In this paper, we investigate the effects of two-step classification and regression on different levels of detection layers and propose a novel face detection framework, named Selective Refinement Network (SRN), which selectively applies two-step classification and regression to specific levels of detection layers. The network structure of SRN is shown in Figure 2, which consists of two key modules, named as the Selective Two-step Classification (STC) module and the Selective Two-step Regression (STR) module. Specifically, the STC is applied to filter out most simple negative samples (illustrated in Figure 1(a)) from the low levels of detection layers, which contains $88.9 \%$ samples. As shown in Figure 1(b), RetinaNet with STC improves the recall efficiency to a certain extent. On the other hand, the design of STR draws on the cascade idea to coarsely adjust the locations and sizes of anchors (illustrated in Figure 1(c)) from high levels of detection layers to provide better initialization for the subsequent regressor. In addition, we design a Receptive Field Enhancement (RFE) to provide more diverse receptive fields to better capture the extreme-pose faces. Extensive experiments have been conducted on AFW, PASCAL face, FDDB, and WIDER FACE benchmarks and we set a new state-ofthe-art performance.

\footnotetext{
${ }^{1}$ http://wider-challenge.org
}

In summarization, we have made the following main contributions to the face detection studies:

- We present a STC module to filter out most simple negative samples from low level layers to reduce the classification search space.

- We design a STR module to coarsely adjust the locations and sizes of anchors from high level layers to provide better initialization for the subsequent regressor.

- We introduce a RFE module to provide more diverse receptive fields for detecting extreme-pose faces.

- We achieve state-of-the-art results on AFW, PASCAL face, FDDB, and WIDER FACE datasets.

\section{Related Work}

Face detection has been a challenging research field since its emergence in the 1990s. Viola and Jones pioneer to use Haar features and AdaBoost to train a face detector with promising accuracy and efficiency (Viola and Jones 2004), which inspires several different approaches afterwards (Liao, Jain, and Li 2016; Brubaker et al. 2008). Apart from those, another important job is the introduction of Deformable Part Model (DPM) (Mathias et al. 2014; Yan et al. 2014a; Zhu and Ramanan 2012).

Recently, face detection has been dominated by the CNNbased methods. CascadeCNN (Li et al. 2015) improves detection accuracy by training a serious of interleaved CNN models and following work (Qin et al. 2016) proposes to jointly train the cascaded CNNs to realize end-to-end optimization. MTCNN (Zhang et al. 2016) proposes a joint face detection and alignment method using multi-task cascaded CNNs. Faceness (Yang et al. 2015) formulates face detection as scoring facial parts responses to detect faces under severe occlusion. UnitBox (Yu et al. 2016) introduces an IoU loss for bounding box prediction. EMO (Zhu et al. 2018) proposes an Expected Max Overlapping score to evaluate the quality of anchor matching. SAFD (Hao et al. 2017) develops a scale proposal stage which automatically normalizes face sizes prior to detection. $S^{2}$ AP (Song et al. 2018) pays attention to specific scales in image pyramid and valid locations in each scales layer. PCN (Shi et al. 2018) proposes a cascade-style structure to rotate faces in a coarse-to-fine manner. Recent work (Bai et al. 2018) designs a novel network to directly generate a clear super-resolution face from a blurry small one.

Additionally, face detection has inherited some achievements from generic object detectors, such as Faster RCNN (Ren et al. 2017), SSD (Liu et al. 2016), FPN (Lin et al. 2017a) and RetinaNet (Lin et al. 2017b). Face RCNN (Wang et al. 2017a) combines Faster R-CNN with hard negative mining and achieves promising results. FaceBoxes (Zhang et al. 2017a) introduces a CPU real-time detecotor based on SSD. Face R-FCN (Wang et al. 2017b) applies R-FCN in face detection and makes according improvements. The face detection model for finding tiny faces (Hu and Ramanan 2017) trains separate detectors for different scales. $S^{3}$ FD (Zhang et al. 2017b) presents multiple strategies onto SSD to compensate for the matching 


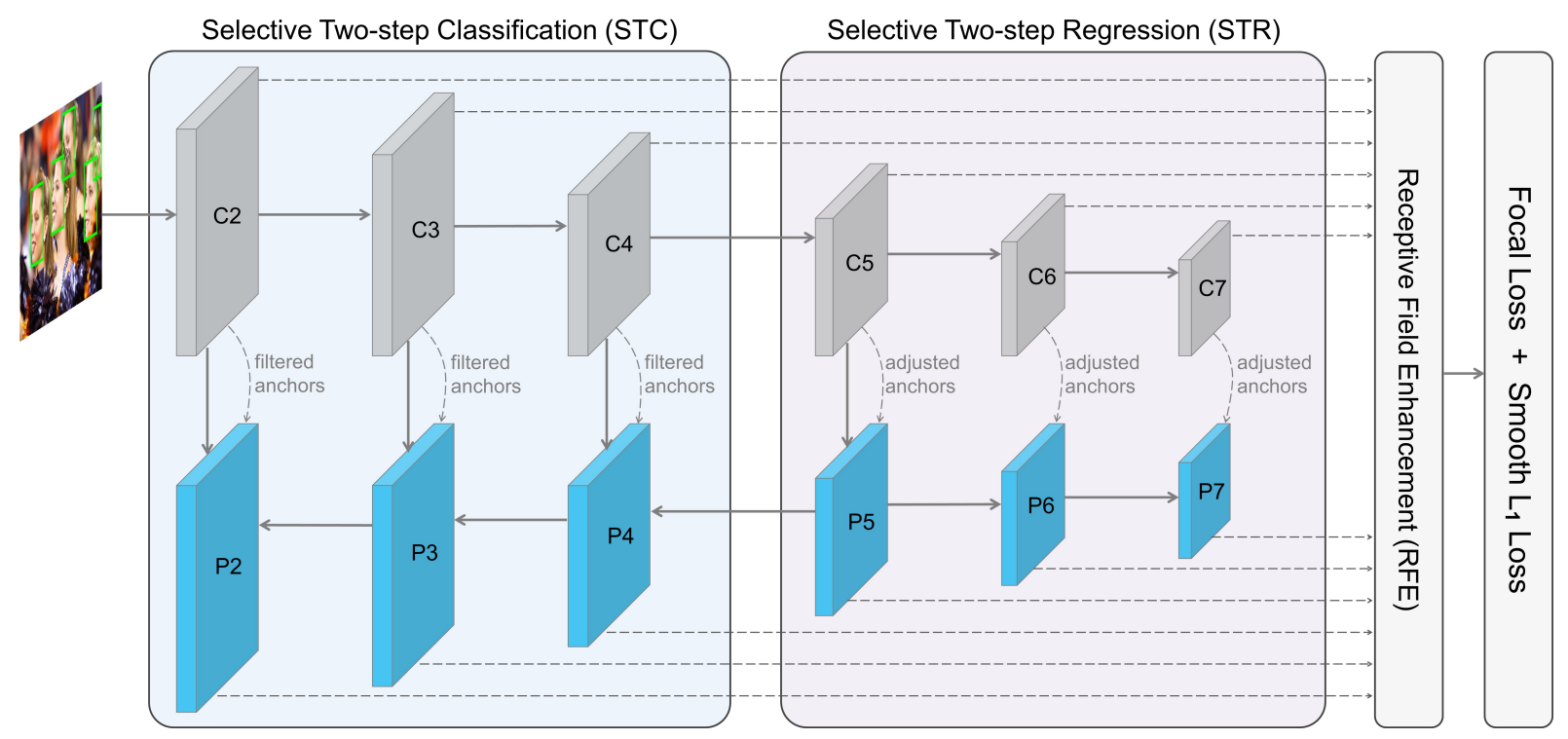

Figure 2: Network structure of SRN. It consists of STC, STR, and RFE. STC uses the first-step classifier to filter out most simple negative anchors from low level detection layers to reduce the search space for the second-step classifier. STR applies the first-step regressor to coarsely adjust the locations and sizes of anchors from high level detection layers to provide better initialization for the second-step regressor. RFE provides more diverse receptive fields to better capture extreme-pose faces.

problem of small faces. SSH (Najibi et al. 2017) models the context information by large filters on each prediction module. PyramidBox (Tang et al. 2018) utilizes contextual information with improved SSD network structure. FAN (Wang, Yuan, and Yu 2017) proposes an anchor-level attention into RetinaNet to detect the occluded faces. In this paper, inspired by the multi-step classification and regression in RefineDet (Zhang et al. 2018) and the focal loss in RetinaNet, we develop a state-of-the-art face detector.

\section{Selective Refinement Network}

\section{Network Structure}

The overall framework of SRN is shown in Figure 2, we describe each component as follows.

Backbone. We adopt ResNet-50 (He et al. 2016) with 6level feature pyramid structure as the backbone network for SRN. The feature maps extracted from those four residual blocks are denoted as $\mathrm{C} 2, \mathrm{C} 3, \mathrm{C} 4$, and $\mathrm{C} 5$, respectively. $\mathrm{C} 6$ and $\mathrm{C} 7$ are just extracted by two simple down-sample $3 \times 3$ convolution layers after $\mathrm{C} 5$. The lateral structure between the bottom-up and the top-down pathways is the same as (Lin et al. 2017a). P2, P3, P4, and P5 are the feature maps extracted from lateral connections, corresponding to C2, C3, $\mathrm{C} 4$, and C5 that are respectively of the same spatial sizes, while $\mathrm{P} 6$ and $\mathrm{P} 7$ are just down-sampled by two $3 \times 3$ convolution layers after P5.

Dedicated Modules. The STC module selects C2, C3, C4, P2, P3, and P4 to perform two-step classification, while the STR module selects C5, C6, C7, P5, P6, and P7 to conduct two-step regression. The RFE module is responsible for enriching the receptive field of features that are used to predict the classification and location of objects.
Anchor Design. At each pyramid level, we use two specific scales of anchors (i.e., $2 S$ and $2 \sqrt{2} S$, where $S$ represents the total stride size of each pyramid level) and one aspect ratios (i.e., 1.25). In total, there are $A=2$ anchors per level and they cover the scale range $8-362$ pixels across levels with respect to the network's input image.

Loss Function. We append a hybrid loss at the end of the deep architecture, which leverage the merits of the focal loss and the smooth $\mathrm{L}_{1}$ loss to drive the model to focus on more hard training examples and learn better regression results.

\section{Selective Two-Step Classification}

Introduced in RefineDet (Zhang et al. 2018), the two-step classification is a kind of cascade classification implemented through a two-step network architecture, in which the first step filters out most simple negative anchors using a preset negative threshold $\theta=0.99$ to reduce the search space for the subsequent step. For anchor-based face detectors, it is necessary to tile plenty of small anchors over the image to detect small faces, which causes the extreme class imbalance between the positive and negative samples. For example, in the SRN structure with the $1024 \times 1024$ input resolution, if we tile 2 anchors at each anchor point, the total number of samples will reach $300 k$. Among them, the number of positive samples is only a few dozen or less. To reduce search space of classifier, it is essential to do two-step classification to reduce the false positives.

However, it is unnecessary to perform two-step classification in all pyramid levels. Since the anchors tiled on the three higher levels (i.e., P5, P6, and P7) only account for $11.1 \%$ and the associated features are much more adequate. Therefore, the classification task is relatively easy in these three higher pyramid levels. It is thus dispensable to apply two- 
step classification on the three higher pyramid levels, and if applied, it will lead to an increase in computation cost. In contrast, the three lower pyramid levels (i.e., P2, P3, and P4) have the vast majority of samples $(88.9 \%)$ and lack of adequate features. It is urgently needed for these low pyramid levels to do two-step classification in order to alleviate the class imbalance problem and reduce the search space for the subsequent classifier.

Therefore, our STC module selects C2, C3, C4, P2, P3, and $\mathrm{P} 4$ to perform two-step classification. As the statistical result shown in Figure 1(a), the STC increases the positive/negative sample ratio by approximately 38 times, from around 1:15441 to $1: 404$. In addition, we use the focal loss in both two steps to make full use of samples. Unlike RefineDet (Zhang et al. 2018), the SRN shares the same classification module in the two steps, since they have the same task to distinguish the face from the background. The experimental results of applying the two-step classification on each pyramid level are shown in Table 2. Consistent with our analysis, the two-step classification on the three lower pyramid levels helps to improve performance, while on the three higher pyramid levels is ineffective.

The loss function for STC consists of two parts, i.e., the loss in the first step and the second step. For the first step, we calculate the focal loss for those samples selected to perform two-step classification. And for the second step, we just focus on those samples that remain after the first step filtering. With these definitions, we define the loss function as:

$$
\begin{aligned}
\mathcal{L}_{\mathrm{STC}}\left(\left\{p_{i}\right\},\left\{q_{i}\right\}\right) & =\frac{1}{N_{\mathrm{s}_{1}}} \sum_{i \in \Omega} \mathcal{L}_{\mathrm{FL}}\left(p_{i}, l_{i}^{*}\right) \\
& +\frac{1}{N_{\mathrm{s}_{2}}} \sum_{i \in \Phi} \mathcal{L}_{\mathrm{FL}}\left(q_{i}, l_{i}^{*}\right),
\end{aligned}
$$

where $i$ is the index of anchor in a mini-batch, $p_{i}$ and $q_{i}$ are the predicted confidence of the anchor $i$ being a face in the first and second steps, $l_{i}^{*}$ is the ground truth class label of anchor $i, N_{\mathrm{s} 1}$ and $N_{\mathrm{s} 2}$ are the numbers of positive anchors in the first and second steps, $\Omega$ represents a collection of samples selected for two-step classification, and $\Phi$ represents a sample set that remains after the first step filtering. The binary classification loss $\mathcal{L}_{\mathrm{FL}}$ is the sigmoid focal loss over two classes (face $v s$. background).

\section{Selective Two-Step Regression}

In the detection task, to make the location of bounding boxes more accurate has always been a challenging problem. Current one-stage methods rely on one-step regression based on various feature layers, which is inaccurate in some challenging scenarios, e.g., MS COCO-style evaluation standard. In recent years, using cascade structure (Zhang et al. 2018; Cai and Vasconcelos 2018) to conduct multi-step regression is an effective method to improve the accuracy of the detection bounding boxes.

However, blindly adding multi-step regression to the specific task (i.e., face detection) is often counterproductive. Experimental results (see Table 4) indicate that applying two-step regression in the three lower pyramid levels impairs the performance. The reasons behind this phenomenon are twofold: 1) the three lower pyramid levels are associated with plenty of small anchors to detect small faces. These small faces are characterized by very coarse feature representations, so it is difficult for these small anchors to perform two-step regression; 2) in the training phase, if we let the network pay too much attention to the difficult regression task on the low pyramid levels, it will cause larger regression loss and hinder the more important classification task.

Based on the above analyses, we selectively perform twostep regression on the three higher pyramid levels. The motivation behind this design is to sufficiently utilize the detailed features of large faces on the three higher pyramid levels to regress more accurate locations of bounding boxes and to make three lower pyramid levels pay more attention to the classification task. This divide-and-conquer strategy makes the whole framework more efficient.

The loss function of STR also consists of two parts, which is shown below:

$$
\begin{aligned}
\mathcal{L}_{\mathrm{STR}}\left(\left\{x_{i}\right\},\left\{t_{i}\right\}\right) & =\sum_{i \in \Psi}\left[l_{i}^{*}=1\right] \mathcal{L}_{\mathrm{r}}\left(x_{i}, g_{i}^{*}\right) \\
& +\sum_{i \in \Phi}\left[l_{i}^{*}=1\right] \mathcal{L}_{\mathrm{r}}\left(t_{i}, g_{i}^{*}\right),
\end{aligned}
$$

where $g_{i}^{*}$ is the ground truth location and size of anchor $i$, $x_{i}$ is the refined coordinates of the anchor $i$ in the first step, $t_{i}$ is the coordinates of the bounding box in the second step, $\Psi$ represents a collection of samples selected for two-step regression, $l_{i}^{*}$ and $\Phi$ are the same as defined in STC. Similar to Faster R-CNN (Ren et al. 2017), we use the smooth $\mathrm{L}_{1}$ loss as the regression loss $L_{\mathrm{r}}$. The Iverson bracket indicator function $\left[l_{i}^{*}=1\right]$ outputs 1 when the condition is true, i.e., $l_{i}^{*}=1$ (the anchor is not the negative), and 0 otherwise. Hence $\left[l_{i}^{*}=1\right] \mathcal{L}_{\mathrm{r}}$ indicates that the regression loss is ignored for negative anchors.

\section{Receptive Field Enhancement}

At present, most detection networks utilize ResNet and VGGNet as the basic feature extraction module, while both of them possess square receptive fields. The singleness of the receptive field affects the detection of objects with different aspect ratios. This issue seems unimportant in face detection task, because the aspect ratio of face annotations is about 1:1 in many datasets. Nevertheless, statistics show that the WIDER FACE training set has a considerable part of faces that have an aspect ratio of more than 2 or less than 0.5 . Consequently, there is a mismatch between the receptive field of network and the aspect ratio of faces.

To address this issue, we propose a module named Receptive Field Enhancement (RFE) to diversify the receptive field of features before predicting classes and locations. In particular, RFE module replaces the middle two convolution layers in the class subnet and the box subnet of RetinaNet. The structure of RFE is shown in Figure 3. Our RFE module adopts a four-branch structure, which is inspired by the Inception block (Szegedy et al. 2015). To be specific, first, we use a $1 \times 1$ convolution layer to decrease the channel number to one quarter of the previous layer. Second, we use $1 \times k$ and $k \times 1$ ( $k=3$ and 5 ) convolution layer to provide rectangular receptive field. Through another $1 \times 1$ convolution 


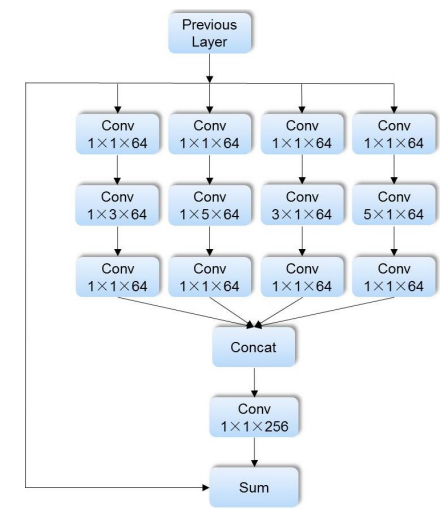

Figure 3: Structure of RFE module.

layer, the feature maps from four branches are concatenated together. Additionally, we apply a shortcut path to retain the original receptive field from previous layer.

\section{Training and Inference}

Training Dataset. All the models are trained on the training set of the WIDER FACE dataset (Yang et al. 2016). It consists of 393, 703 annotated face bounding boxes in 32, 203 images with variations in pose, scale, facial expression, occlusion, and lighting condition. The dataset is split into the training (40\%), validation (10\%) and testing (50\%) sets, and defines three levels of difficulty: Easy, Medium, Hard, based on the detection rate of EdgeBox (Zitnick and Dollár 2014).

Data Augmentation. To prevent over-fitting and construct a robust model, several data augmentation strategies are used to adapt to face variations, described as follows.

1) Applying some photometric distortions introduced in previous work (Howard 2013) to the training images.

2) Expanding the images with a random factor in the interval $[1,2]$ by the zero-padding operation.

3) Cropping two square patches and randomly selecting one for training. One patch is with the size of the image's shorter side and the other one is with the size determined by multiplying a random number in the interval $[0.5,1.0]$ by the image's shorter side.

4) Flipping the selected patch randomly and resizing it to $1024 \times 1024$ to get the final training sample.

Anchor Matching. During the training phase, anchors need to be divided into positive and negative samples. Specifically, anchors are assigned to ground-truth face boxes using an intersection-over-union (IoU) threshold of $\theta_{p}$; and to background if their IoU is in $\left[0, \theta_{n}\right)$. If an anchor is unassigned, which may happen with overlap in $\left[\theta_{n}, \theta_{p}\right)$, it is ignored during training. Empirically, we set $\theta_{n}=0.3$ and $\theta_{p}=0.7$ for the first step, and $\theta_{n}=0.4$ and $\theta_{p}=0.5$ for the second step.

Optimization. The loss function for SRN is just the sum of the STC loss and the STR loss, i.e., $\mathcal{L}=\mathcal{L}_{\mathrm{STC}}+\mathcal{L}_{\mathrm{STR}}$. The backbone network is initialized by the pretrained ResNet50 model (Russakovsky et al. 2015) and all the parameters in the newly added convolution layers are initialized by the "xavier" method. We fine-tune the SRN model using SGD with 0.9 momentum, 0.0001 weight decay, and batch size 32 . We set the learning rate to $10^{-2}$ for the first 100 epochs, and decay it to $10^{-3}$ and $10^{-4}$ for another 20 and 10 epochs, respectively. We implement SRN using the PyTorch library (Paszke et al. 2017).

Inference. In the inference phase, the STC first filters the regularly tiled anchors on the selected pyramid levels with the negative confidence scores larger than the threshold $\theta=0.99$, and then STR adjusts the locations and sizes of selected anchors. After that, the second step takes over these refined anchors, and outputs top 2000 high confident detections. Finally, we apply the non-maximum suppression (NMS) with jaccard overlap of 0.5 to generate the top 750 high confident detections per image as the final results.

\section{Experiments}

We first analyze the proposed method in detail to verify the effectiveness of our contributions. Then we evaluate the final model on the common face detection benchmark datasets, including AFW (Zhu and Ramanan 2012), PASCAL Face (Yan et al. 2014b), FDDB (Jain and Learned-Miller 2010), and WIDER FACE (Yang et al. 2016).

\section{Model Analysis}

We conduct a set of ablation experiments on the WIDER FACE dataset to analyze our model in detail. For a fair comparison, we use the same parameter settings for all the experiments, except for specified changes to the components. All models are trained on the WIDER FACE training set and evaluated on the validation set.

Ablation Setting. To better understand SRN, we ablate each component one after another to examine how each proposed component affects the final performance. Firstly, we use the ordinary prediction head in (Lin et al. 2017b) instead of the proposed RFE. Secondly, we ablate the STR or STC module to verify their effectiveness. The results of ablation experiments are listed in Table 1 and some promising conclusions can be drawn as follows.

Table 1: Effectiveness of various designs on the AP performance.

\begin{tabular}{|c|c|c|c|c|c|}
\hline Component & \multicolumn{4}{|c|}{ SRN } & \\
\hline STC & & $\sqrt{ }$ & & $\sqrt{ }$ & $\sqrt{ }$ \\
\hline STR & & & $\checkmark$ & $\sqrt{ }$ & $\sqrt{ }$ \\
\hline RFE & & & & & $\checkmark$ \\
\hline Easy subset & 95.1 & 95.3 & 95.9 & 96.1 & 96.4 \\
\hline Medium subset & 93.9 & 94.4 & 94.8 & 95.0 & 95.3 \\
\hline Hard subset & 88.0 & 89.4 & 88.8 & 90.1 & 90.2 \\
\hline
\end{tabular}

Selective Two-step Classification. Experimental results of applying two-step classification to each pyramid level are 


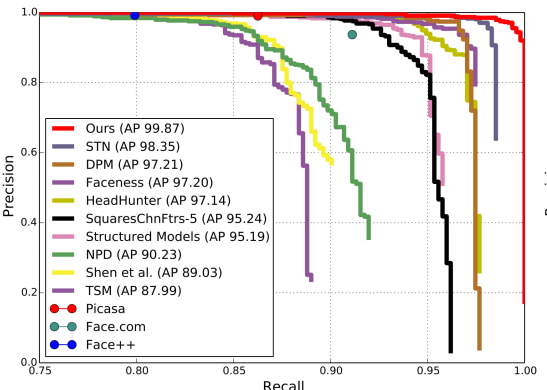

(a) AFW

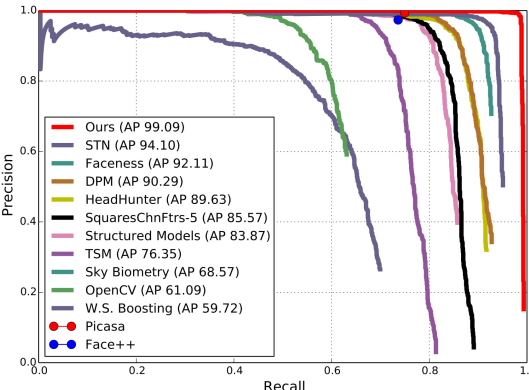

(b) PASCAL face

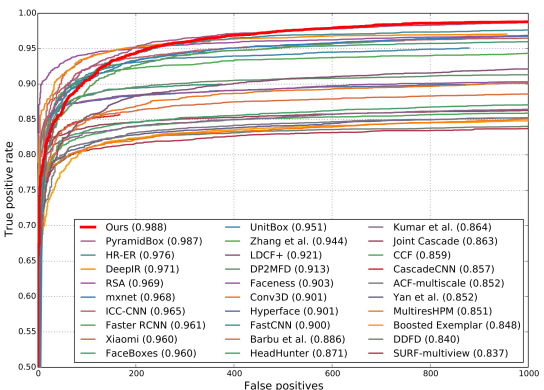

(c) FDDB

Figure 4: Evaluation on the common face detection datasets.

shown in Table 2, indicating that applying two-step classification to the low pyramid levels improves the performance, especially on tiny faces. Therefore, the STC module selectively applies the two-step classification on the low pyramid levels (i.e., P2, P3, and P4), since these levels are associated with lots of small anchors, which are the main source of false positives. As shown in Table 1, we find that after using the STC module, the AP scores of the detector are improved from $95.1 \%, 93.9 \%$ and $88.0 \%$ to $95.3 \%, 94.4 \%$ and $89.4 \%$ on the Easy, Medium and Hard subsets, respectively. In order to verify whether the improvements benefit from reducing the false positives, we count the number of false positives under different recall rates. As listed in Table 3, our STC effectively reduces the false positives across different recall rates, demonstrating the effectiveness of the STC module.

Table 2: AP performance of the two-step classification applied to each pyramid level.

\begin{tabular}{c|c|cccccc}
\hline STC & B & P2 & P3 & P4 & P5 & P6 & P7 \\
\hline Easy & 95.1 & $\mathbf{9 5 . 2}$ & $\mathbf{9 5 . 2}$ & $\mathbf{9 5 . 2}$ & 95.0 & 95.1 & 95.0 \\
Medium & 93.9 & $\mathbf{9 4 . 2}$ & $\mathbf{9 4 . 3}$ & $\mathbf{9 4 . 1}$ & 93.9 & 93.7 & 93.9 \\
Hard & 88.0 & $\mathbf{8 8 . 9}$ & $\mathbf{8 8 . 7}$ & $\mathbf{8 8 . 5}$ & 87.8 & 88.0 & 87.7 \\
\hline
\end{tabular}

Table 3: Number of false positives at different recall rates.

\begin{tabular}{c|cccccc}
\hline Recall (\%) & 10 & 30 & 50 & 80 & 90 & 95 \\
\hline \# FP of RetinaNet & 3 & 24 & 126 & 2801 & 27644 & 466534 \\
\# FP of SRN (STC only) & 1 & 20 & 101 & 2124 & 13163 & 103586 \\
\hline
\end{tabular}

Selective Two-step Regression. We only add the STR module to our baseline detector to verify its effectiveness. As shown in Table 1, it produces much better results than the baseline, with $0.8 \%, 0.9 \%$ and $0.8 \%$ AP improvements on the Easy, Medium, and Hard subsets. Experimental results of applying two-step regression to each pyramid level (see Table 4) confirm our previous analysis. Inspired by the detection evaluation metric of MS COCO, we use 4 IoU thresholds $\{0.5,0.6,0.7,0.8\}$ to compute the AP, so as to prove that the STR module can produce more accurate localization. As shown in Table 5, the STR module produces consistently accurate detection results than the baseline method.
The gap between the AP across all three subsets increases as the IoU threshold increases, which indicate that the STR module is important to produce more accurate detections. In addition, coupled with the STC module, the performance is further improved to $96.1 \%, 95.0 \%$ and $90.1 \%$ on the Easy, Medium and Hard subsets, respectively.

Table 4: AP performance of the two-step regression applied to each pyramid level.

\begin{tabular}{c|c|cccccc}
\hline STR & B & P2 & P3 & P4 & P5 & P6 & P7 \\
\hline Easy & 95.1 & 94.8 & 94.3 & 94.8 & $\mathbf{9 5 . 4}$ & $\mathbf{9 5 . 7}$ & $\mathbf{9 5 . 6}$ \\
Medium & 93.9 & 93.4 & 93.7 & 93.9 & $\mathbf{9 4 . 2}$ & $\mathbf{9 4 . 4}$ & $\mathbf{9 4 . 6}$ \\
Hard & 88.0 & 87.5 & 87.7 & 87.0 & $\mathbf{8 8 . 2}$ & $\mathbf{8 8 . 2}$ & $\mathbf{8 8 . 4}$ \\
\hline
\end{tabular}

Table 5: AP at different IoU thresholds on the WIDER FACE Hard subset.

\begin{tabular}{c|cccc}
\hline IoU & 0.5 & 0.6 & 0.7 & 0.8 \\
\hline RetinaNet & 88.1 & 76.4 & 57.8 & 28.5 \\
SRN (STR only) & 88.8 & 83.4 & 66.5 & 38.2 \\
\hline
\end{tabular}

Receptive Field Enhancement. The RFE is used to diversify the receptive fields of detection layers in order to capture faces with extreme poses. Comparing the detection results between fourth and fifth columns in Table 1, we notice that RFE consistently improves the AP scores in different subsets, i.e., $0.3 \%, 0.3 \%$, and $0.1 \%$ APs on the Easy, Medium, and Hard categories. These improvements can be mainly attributed to the diverse receptive fields, which is useful to capture various pose faces for better detection accuracy.

\section{Evaluation on Benchmark}

AFW Dataset. It consists of 205 images with 473 labeled faces. The images in the dataset contain cluttered backgrounds with large variations in both face viewpoint and appearance. We compare SRN against seven state-ofthe-art methods and three commercial face detectors (i.e., Face.com, Face++ and Picasa). As shown in Figure 4(a), SRN outperforms these state-of-the-art methods with the top AP score (99.87\%). 


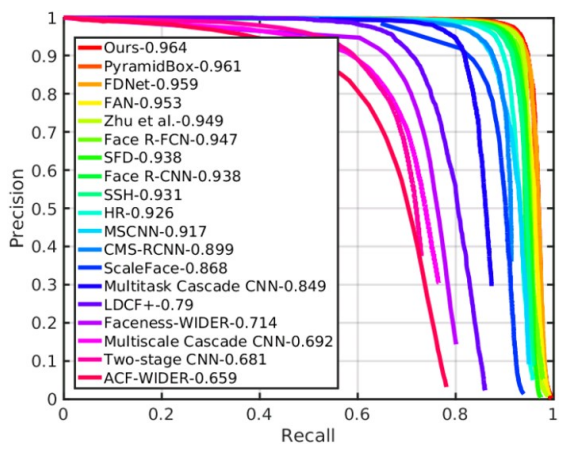

(a) Val: Easy

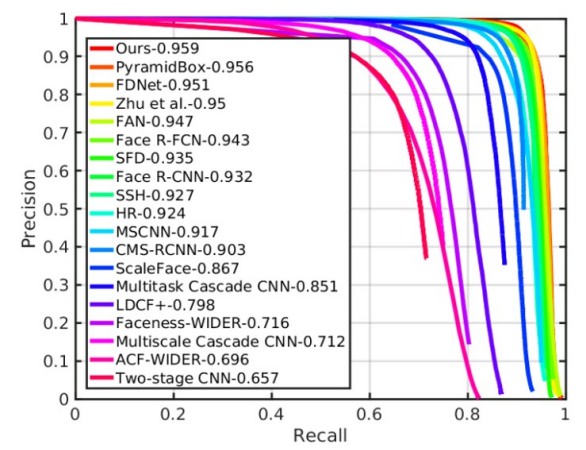

(d) Test: Easy

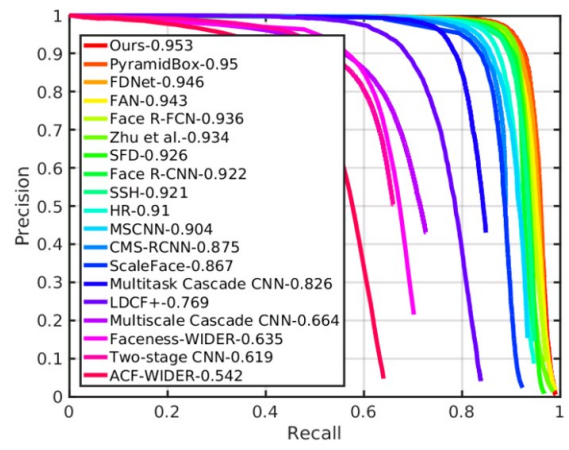

(b) Val: Medium

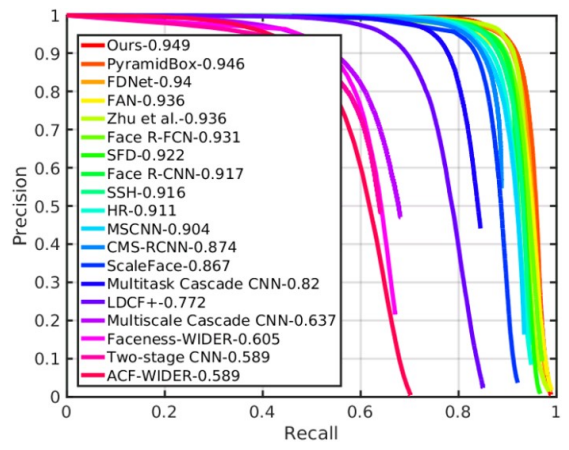

(e) Test: Medium

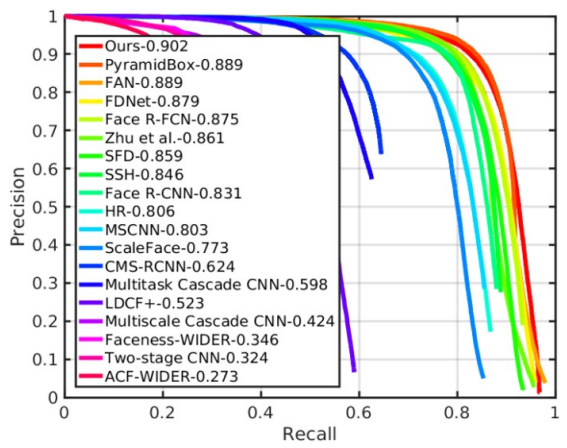

(c) Val: Hard

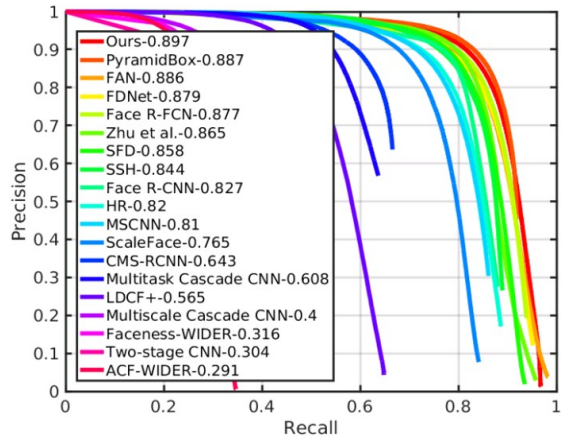

(f) Test: Hard

Figure 5: Precision-recall curves on WIDER FACE validation and testing subsets.

PASCAL Face Dataset. It has 1,335 labeled faces in 851 images with large face appearance and pose variations. We present the precision-recall curves of the proposed SRN method and six state-of-the-art methods and three commercial face detectors (i.e., SkyBiometry, Face++ and Picasa) in Figure 4(b). SRN achieves the state-of-the-art results by improving $4.99 \%$ AP score compared to the second best method STN (Chen et al. 2016).

FDDB Dataset. It contains 5, 171 faces annotated in 2, 845 images with a wide range of difficulties, such as occlusions, difficult poses, and low image resolutions. We evaluate the proposed SRN detector on the FDDB dataset and compare it with several state-of-the-art methods. As shown in Figure 4(c), our SRN sets a new state-of-the-art performance, i.e., 98.8\% true positive rate when the number of false positives is equal to 1000 . These results indicate that SRN is robust to varying scales, large appearance changes, heavy occlusions, and severe blur degradations that are prevalent in detecting face in unconstrained real-life scenarios.

WIDER FACE Dataset. We compare SRN with eighteen state-of-the-art face detection methods on both the validation and testing sets. To obtain the evaluation results on the testing set, we submit the detection results of SRN to the authors for evaluation. As shown in Figure 5, we find that SRN performs favourably against the state-of-the-art based on the average precision (AP) across the three subsets, especially on the Hard subset which contains a large amount of small faces. Specifically, it produces the best AP scores in all subsets of both validation and testing sets, i.e., 96.4\% (Easy), $95.3 \%$ (Medium) and $90.2 \%$ (Hard) for validation set, and
95.9\% (Easy), 94.9\% (Medium) and $89.7 \%$ (Hard) for testing set, surpassing all approaches, which demonstrates the superiority of the proposed detector.

\section{Conclusion}

In this paper, we have presented SRN, a novel single shot face detector, which consists of two key modules, i.e., the STC and the STR. The STC uses the first-step classifier to filter out most simple negative anchors from low level detection layers to reduce the search space for the second-step classifier, so as to reduce false positives. And the STR applies the first-step regressor to coarsely adjust the locations and sizes of anchors from high level detection layers to provide better initialization for the second-step regressor, in order to improve the location accuracy of bounding boxes. Moreover, the RFE is introduced to provide diverse receptive fields to better capture faces in some extreme poses. Extensive experiments on the AFW, PASCAL face, FDDB and WIDER FACE datasets demonstrate that SRN achieves the state-of-the-art detection performance.

\section{Acknowledgement}

The work is supported by the Young Thousand Talents Program, the Natural Science Foundation of China (Grant No.61672519, No.61876178), the research project from Huawei Inc. (Grant No. YBN2018065193), and the independent research project of National Laboratory of Pattern Recognition. 


\section{References}

Bai, Y.; Zhang, Y.; Ding, M.; and Ghanem, B. 2018. Finding tiny faces in the wild with generative adversarial network. In CVPR.

Brubaker, S. C.; Wu, J.; Sun, J.; Mullin, M. D.; and Rehg, J. M. 2008. On the design of cascades of boosted ensembles for face detection. IJCV.

Cai, Z., and Vasconcelos, N. 2018. Cascade R-CNN: delving into high quality object detection. In $C V P R$.

Chen, D.; Hua, G.; Wen, F.; and Sun, J. 2016. Supervised transformer network for efficient face detection. In $E C C V$.

Gidaris, S., and Komodakis, N. 2015. Object detection via a multi-region and semantic segmentation-aware CNN model. In $I C C V$.

Girshick, R. B. 2015. Fast R-CNN. In ICCV.

Hao, Z.; Liu, Y.; Qin, H.; Yan, J.; Li, X.; and Hu, X. 2017. Scale-aware face detection. In $C V P R$.

He, K.; Zhang, X.; Ren, S.; and Sun, J. 2016. Deep residual learning for image recognition. In $C V P R$.

Howard, A. G. 2013. Some improvements on deep convolutional neural network based image classification. CoRR.

Hu, P., and Ramanan, D. 2017. Finding tiny faces. In CVPR. Jain, V., and Learned-Miller, E. 2010. Fddb: A benchmark for face detection in unconstrained settings. Technical report, University of Massachusetts, Amherst.

Li, H.; Lin, Z.; Shen, X.; Brandt, J.; and Hua, G. 2015. A convolutional neural network cascade for face detection. In CVPR.

Liao, S.; Jain, A. K.; and Li, S. Z. 2016. A fast and accurate unconstrained face detector. TPAMI.

Lin, T.; Maire, M.; Belongie, S. J.; Hays, J.; Perona, P.; Ramanan, D.; Dollár, P.; and Zitnick, C. L. 2014. Microsoft COCO: common objects in context. In ECCV.

Lin, T.; Dollár, P.; Girshick, R. B.; He, K.; Hariharan, B.; and Belongie, S. J. 2017a. Feature pyramid networks for object detection. In CVPR.

Lin, T.; Goyal, P.; Girshick, R. B.; He, K.; and Dollár, P. 2017b. Focal loss for dense object detection. In ICCV.

Liu, W.; Anguelov, D.; Erhan, D.; Szegedy, C.; Reed, S. E.; Fu, C.; and Berg, A. C. 2016. SSD: single shot multibox detector. In $E C C V$.

Mathias, M.; Benenson, R.; Pedersoli, M.; and Gool, L. J. V. 2014. Face detection without bells and whistles. In ECCV.

Najibi, M.; Samangouei, P.; Chellappa, R.; and Davis, L. S. 2017. SSH: single stage headless face detector. In ICCV.

Paszke, A.; Gross, S.; Chintala, S.; and Chanan, G. 2017. Pytorch.

Qin, H.; Yan, J.; Li, X.; and Hu, X. 2016. Joint training of cascaded CNN for face detection. In CVPR.

Ren, S.; He, K.; Girshick, R. B.; and Sun, J. 2017. Faster $\mathrm{R}-\mathrm{CNN}$ : towards real-time object detection with region proposal networks. TPAMI.
Russakovsky, O.; Deng, J.; Su, H.; Krause, J.; Satheesh, S.; Ma, S.; Huang, Z.; Karpathy, A.; Khosla, A.; Bernstein, M. S.; Berg, A. C.; and Li, F. 2015. Imagenet large scale visual recognition challenge. IJCV.

Shi, X.; Shan, S.; Kan, M.; Wu, S.; and Chen, X. 2018. Real-time rotation-invariant face detection with progressive calibration networks. In $C V P R$.

Song, G.; Liu, Y.; Jiang, M.; Wang, Y.; Yan, J.; and Leng, B. 2018. Beyond trade-off: Accelerate fcn-based face detector with higher accuracy. In $C V P R$.

Szegedy, C.; Liu, W.; Jia, Y.; Sermanet, P.; Reed, S. E.; Anguelov, D.; Erhan, D.; Vanhoucke, V.; and Rabinovich, A. 2015. Going deeper with convolutions. In CVPR.

Tang, X.; Du, D. K.; He, Z.; and Liu, J. 2018. Pyramidbox: A context-assisted single shot face detector. In ECCV.

Viola, P. A., and Jones, M. J. 2004. Robust real-time face detection. IJCV.

Wang, H.; Li, Z.; Ji, X.; and Wang, Y. 2017a. Face r-cnn. CoRR.

Wang, Y.; Ji, X.; Zhou, Z.; Wang, H.; and Li, Z. 2017 b. Detecting faces using region-based fully convolutional networks. CoRR.

Wang, J.; Yuan, Y.; and Yu, G. 2017. Face attention network: An effective face detector for the occluded faces. CoRR.

Yan, J.; Lei, Z.; Wen, L.; and Li, S. Z. 2014a. The fastest deformable part model for object detection. In CVPR.

Yan, J.; Zhang, X.; Lei, Z.; and Li, S. Z. 2014b. Face detection by structural models. IVC.

Yang, S.; Luo, P.; Loy, C. C.; and Tang, X. 2015. From facial parts responses to face detection: A deep learning approach. In $I C C V$.

Yang, S.; Luo, P.; Loy, C. C.; and Tang, X. 2016. WIDER FACE: A face detection benchmark. In CVPR.

Yu, J.; Jiang, Y.; Wang, Z.; Cao, Z.; and Huang, T. S. 2016. Unitbox: An advanced object detection network. In ACMMM.

Zhang, K.; Zhang, Z.; Li, Z.; and Qiao, Y. 2016. Joint face detection and alignment using multitask cascaded convolutional networks. SPL.

Zhang, S.; Zhu, X.; Lei, Z.; Shi, H.; Wang, X.; and Li, S. Z. 2017a. Faceboxes: A CPU real-time face detector with high accuracy. In $I J C B$.

Zhang, S.; Zhu, X.; Lei, Z.; Shi, H.; Wang, X.; and Li, S. Z. 2017b. $S^{3}$ FD: Single shot scale-invariant face detector. In $I C C V$.

Zhang, S.; Wen, L.; Bian, X.; Lei, Z.; and Li, S. Z. 2018. Single-shot refinement neural network for object detection. In CVPR.

Zhu, X., and Ramanan, D. 2012. Face detection, pose estimation, and landmark localization in the wild. In CVPR.

Zhu, C.; Tao, R.; Luu, K.; and Savvides, M. 2018. Seeing small faces from robust anchor's perspective. In CVPR.

Zitnick, C. L., and Dollár, P. 2014. Edge boxes: Locating object proposals from edges. In $E C C V$. 